\title{
Linearly constrained minimum variance spatial filtering for localization of conductivity changes in electrical impedance tomography
}

\author{
M. Fernández-Corazza ${ }^{1,2,3, *, \dagger}$, N. von Ellenrieder ${ }^{1,2}$ and C. H. Muravchik ${ }^{1,4}$ \\ ${ }^{1}$ Laboratorio de Electrónica Industrial, Control e Instrumentación (LEICI), Facultad de Ingeniería Universidad \\ Nacional de La Plata (UNLP), La Plata, Argentina \\ ${ }^{2}$ Consejo Nacional de Investigaciones Científicas y Técnicas (CONICET), Buenos Aires, Argentina \\ ${ }^{3}$ Departamento de Ciencias Básicas, Facultad de Ingeniería, Universidad Nacional de La Plata (UNLP), La \\ Plata, Argentina \\ ${ }^{4}$ Comisión de Investigaciones Científicas de la Prov. de Buenos Aires (CIC-PBA), La Plata, Argentina
}

\begin{abstract}
SUMMARY
We localize dynamic electrical conductivity changes and reconstruct their time evolution introducing the spatial filtering technique to electrical impedance tomography (EIT). More precisely, we use the unit-noisegain constrained variation of the distortionless-response linearly constrained minimum variance spatial filter. We address the effects of interference and the use of zero gain constraints. The approach is successfully tested in simulated and real tank phantoms. We compute the position error and resolution to compare the localization performance of the proposed method with the one-step Gauss-Newton reconstruction with Laplacian prior. We also study the effects of sensor position errors. Our results show that EIT spatial filtering is useful for localizing conductivity changes of relatively small size and for estimating their time-courses. Some potential dynamic EIT applications such as acute ischemic stroke detection and neuronal activity localization may benefit from the higher resolution of spatial filters as compared to conventional tomographic reconstruction algorithms. Copyright (C) 2015 John Wiley \& Sons, Ltd.
\end{abstract}

Received 21 July 2014; Revised 12 January 2015; Accepted 14 January 2015

KEY WORDS: electrical impedance tomography; spatial filtering; linearly constrained minimum variance spatial filter; localization of conductivity changes

\section{INTRODUCTION}

We propose a spatial filtering (SF) technique to localize electrical conductivity changes and to reconstruct their time evolution using electrical impedance tomography (EIT) measurements. EIT is a technique that can be used to reconstruct the internal conductivity map of living tissues. A low electric current is applied to a tissular object originating an electric potential distribution that is sampled with an array of electrodes on the object's surface. The internal conductivity map is obtained from these measurements by means of appropriate reconstruction algorithms. EIT is considered to have a great potential for clinical use because it is relatively safe, minimally invasive, comparatively inexpensive, and portable [1]. When compared with functional magnetic resonance imaging, EIT has a good temporal resolution but a poor spatial resolution. Presently, applications include breast cancer detection [2], lung function monitoring [1], acute stroke detection [3], and brain imaging [4-8]. Although our main motivations are biomedical applications of EIT-SF in the human brain,

\footnotetext{
*Correspondence to: M. Fernández-Corazza, Laboratorio de Electrónica Industrial, Control e Instrumentación (LEICI), Facultad de Ingeniería, Universidad Nacional de La Plata (UNLP), Calle 48 y 116, CC91 (1900), Buenos Aires, Argentina.

${ }^{\dagger}$ E-mail: marianof.corazza@ing.unlp.edu.ar
} 
we present here the details of the method as a more general tool for detecting and characterizing localized conductivity changes inside tissular objects.

Spatial filtering is being widely used in communications, and in the 1990s, it was successfully introduced to the source localization problem in electroencephalography (EEG) and magnetoencephalography (MEG) [9]. A spatial filter is often designed so that the maximum gain of the array is oriented to a desired point in space. The pointing direction is controlled by an appropriate combination of the individual signals measured with each sensor. The output of the filter is used to build an estimate of the signal generated at a specific spatial location. When applied to EEG/MEG data, a virtual scan of the head is usually performed resulting in an activation map of the whole brain or the region of interest. The linearly constrained minimum variance (LCMV) beamformer is a particular spatial filter based on the minimization of the output signal variance under particular constraints [10]. Variations arise from including the information of the source orientation, whether it is assumed to be known or estimated $[9,11,12]$.

However, the application of SF to EIT has not been fully exploited yet. Commonly used EIT algorithms are based either on tomographic-imaging methods with a linear approximation and using a reference or baseline image [13-16] or in nonlinear absolute imaging [17, 18]. In EIT, each snapshot is usually solved independently, resulting in one image per snapshot or time frame. Other approaches considering temporal correlation have been proposed such as the Kalman filter and the temporal reconstruction method $[19,20]$. In the former, the actual conductivity estimate is based on the measurements and on the previous estimate, whereas the latter considers data from adjacent time frames. The LCMV filter studied here generates a single activation map with the information of all the snapshots within a time segment. In a preliminary study, we introduced a technique that allowed to accurately estimate the position of a conductivity change in the brain with a spatial filter [21]. Some potential applications of EIT such as tumor, neuronal activity, epileptic foci, and stroke localization may benefit from the low bias and increased resolution of SF, when compared with other conventional reconstruction algorithms. Moreover, the time-course of localized conductivity changes is also available from SF providing a potential new tool for diagnostic.

In this work, we propose to apply the LCMV filter to EIT, with emphasis on the combination of different electrode pairs for the current injection. We consider localized conductivity changes as 'sources' and use the LCMV filter to estimate their central position and track their time-courses, based on EIT measurements. We extend our preliminary results [21, 22] to include dynamic conductivity changes, to analyze the use of zero gain constraints, to propose an alternative when the conductivity change time-course of interest is previously known, and to evaluate the effects of sensor position errors. We analyze the performance of the algorithm for one-source and two-source scenarios, with focus on the bias, spatial resolution, and quality of the reconstructed time-course. We compare the localization performance of the EIT-SF with the one-step Gauss-Newton reconstruction with Laplacian prior (GN-Laplace) [14]. For two simultaneous and somewhat correlated conductivity changes at different positions, we also analyze how both varying conductivity regions affect each other and how to possibly reduce the leakage in the time-course reconstruction. The LCMV filter performance is evaluated by means of simulated EIT measurements and real EIT measurements on a cylindrical tank phantom.

\section{METHODS}

In EIT, an electric current is applied to an object, and the resulting electric potential is measured at its boundary. The electric potential distribution is a function of the internal conductivity of the object. We describe the model and the formulation of EIT-SF, including the addition of null-gain constraints and known time-courses.

\subsection{Signal model}

Two out of $L$ electrodes are used for current injection, while $L-2$ measure the electric potential. Several reference potential schemes are possible; we adopted an average reference from the $L-2$ measurements. The current injection pair can be chosen among the $L(L-1) / 2$ possible pairs of 
electrodes, according to a measurement protocol. We assume that the pairs are switched fast enough so that the conductivity change between the samples obtained with different pairs is negligible. Thus, for each snapshot $t$, the measurements of all used injection pairs $M$ are arranged into a vector $\boldsymbol{m}_{t}$ of $M(L-2)$ elements.

The measured signals $\boldsymbol{m}_{t}$ can be modeled as a nonlinear function $f$ of the internal conductivity of the object $\boldsymbol{\sigma}_{t}$ plus a noise term $\boldsymbol{n}_{t}$ :

$$
\boldsymbol{m}_{t}=f\left(\sigma_{t}\right)+\boldsymbol{n}_{t} .
$$

The noise term is seen as a zero-mean white Gaussian noise (WGN) modeling the electronic noise because of the contact sensors and the amplifiers and other contributions due to aspects not included in the model. The frequency and phase of the current injection waveform are arbitrary but known. Then, other sources of electromagnetic noise, for example, neuronal population electrical activity if the object is the head, can be neglected if a sufficient number of time samples is available $[23,24]$. The computation of $f\left(\sigma_{t}\right)$, assuming that the conductivity distribution is known, is called the EIT forward problem (FP). The electrical conductivity can be modeled as a continuous tensor in the object, but in practice, the volume is tessellated into a discrete $K$ element domain. Assuming piecewise homogeneity and isotropy, the conductivity at each element is a scalar value. With these assumptions, $\sigma_{t}$ is a vector of length $K$. An approximation to (1) is to linearize the problem around a conductivity baseline distribution $\sigma_{i}$ :

$$
\boldsymbol{m}_{t}=f\left(\sigma_{i}\right)+\boldsymbol{J}\left(\boldsymbol{\sigma}_{t}-\boldsymbol{\sigma}_{i}\right)+\boldsymbol{n}_{t},
$$

where $\boldsymbol{J}=\left[\boldsymbol{j}_{1} \boldsymbol{j}_{2}, \ldots, \boldsymbol{j}_{K}\right]$ is the $M(L-2) \times K$ Jacobian, also called the sensitivity matrix. The column $\boldsymbol{j}_{k}$ of $\boldsymbol{J}$ is the derivative of the potential with respect to the conductivity at the $k$ th element, known as the $k$ th sensitivity vector. In EIT, there are different methods to compute this matrix [16], either analytically for regular volumes [25-27] or numerically using the finite element method (FEM) or the boundary element method [23, 28, 29]. In a previous research, we introduced a new way to compute $\boldsymbol{J}$ based on the mathematical equivalence difference between EIT and the EEG FP $[16,21]$. It needs the computation of only $3 K$ EEG FP solutions to build $\boldsymbol{J}$ for all possible injection pairs. The EEG FP solution estimates the potential at the $L$ electrodes when a dipolar current density source is located at position $k$ and is obtained with FEM. The main advantage of this approach is that only the EEG Jacobian matrix of size $L \times 3 K$ plus the gradient of the potential for each current injection pair $(M \times 3 K)$ must be computed and stored in memory instead of the much larger EIT Jacobian matrix of size $M(L-2) \times K$. The procedure is detailed in Appendix A.

Assuming a conductivity change $\delta \sigma_{k, t}$ at position $k$, the difference between the measurements before $\left(\boldsymbol{m}_{t}\right)$ and after it $\left(\boldsymbol{m}_{i}\right)$ is

$$
\boldsymbol{m}_{t}-\boldsymbol{m}_{i}=\boldsymbol{y}_{t}=\boldsymbol{J}\left(\sigma_{t}-\sigma_{i}\right)+\delta n_{t},
$$

where the $k$ th element of $\left(\sigma_{t}-\sigma_{i}\right)$ is the only nonzero value. Thus,

$$
\boldsymbol{y}_{t}=\boldsymbol{j}_{k} \delta \sigma_{k, t}+\boldsymbol{\delta} \boldsymbol{n}_{t} .
$$

The snapshots $\boldsymbol{y}_{t}, t=1, \ldots, T$ are arranged into a matrix $\boldsymbol{Y}=\left[\boldsymbol{y}_{1}, \ldots, \boldsymbol{y}_{T}\right]$ and similarly with $\boldsymbol{\delta} \boldsymbol{n}_{t}$ into the matrix $\boldsymbol{\delta} \boldsymbol{N}$. The signal model becomes

$$
\boldsymbol{Y}=\boldsymbol{j}_{k} \delta \sigma_{k}^{T}+\boldsymbol{\delta} \boldsymbol{N}
$$

where $\delta \sigma_{k}$ contains the time samples of the conductivity change at position $k$. Note that the sum of the elements of each column of $\boldsymbol{Y}$ is zero because we used an average reference. $\boldsymbol{\delta} \boldsymbol{N}$ also includes other possible conductivity changes unrelated to the one under study, which we also model as WGN.

\subsection{The linearly constrained minimum variance filter}

In this section, we obtain the LCMV filter for EIT measurements, following ideas for brain imaging in EEG/MEG [9-12]. 
The LCMV spatial filter proposes a weight vector $\boldsymbol{w}_{k}$ such that $\boldsymbol{w}_{k}^{T} \boldsymbol{Y}$ is an estimator of the parameter of interest at position $k$, with minimum variance, and subject to particular constraints. In EEG/MEG, the parameters of interest are related to the source of activity, but in EIT, they are related to the conductivity change $\delta \sigma_{k}$. This is why sometimes we refer to the localized conductivity change as a source, although a conductivity change is not in itself an electromagnetic source of activity. According to the distortionless response variation of the LCMV filter, the vectors $\boldsymbol{w}_{k}$ must satisfy

$$
\begin{array}{r}
\underset{\boldsymbol{w}_{k}}{\operatorname{argmin}}\left\{\boldsymbol{w}_{k}^{T} \boldsymbol{C}_{\mathbf{Y}} \boldsymbol{w}_{k}\right\} \\
\text { subject to } \boldsymbol{w}_{k}^{T} \boldsymbol{j}_{k}=1,
\end{array}
$$

where $\boldsymbol{C}_{\mathbf{Y}}=\mathbb{E}\left\{(\boldsymbol{Y}-\mathbb{E}\{\boldsymbol{Y}\})(\boldsymbol{Y}-\mathbb{E}\{\boldsymbol{Y}\})^{T}\right\}$ is the covariance matrix of $\boldsymbol{Y}$, and $\mathbb{E}$ denotes expectation [9]. Following the standard procedure to solve the minimization problem (6), the weight vector for the distortionless response LCMV filter is

$$
\boldsymbol{w}_{k}^{T}=\left(\boldsymbol{j}_{k}^{T} \boldsymbol{C}_{\mathbf{Y}}^{-1} \boldsymbol{j}_{k}\right)^{-1} \boldsymbol{j}_{k}^{T} \boldsymbol{C}_{\mathbf{Y}}^{-1} .
$$

The output of the filter is an estimator of the conductivity change at position $k$,

$$
\widehat{\delta \sigma_{k}^{T}}=w_{k}^{T} \boldsymbol{Y}=\left(j_{k}^{T} C_{\mathbf{Y}}^{-1} j_{k}\right)^{-1} j_{k}^{T} C_{\mathbf{Y}}^{-1} \boldsymbol{Y}
$$

It can be demonstrated that the output of the filter is unbiased for one localized source [9]. Moreover, it is empirically shown that the filter automatically forces null sensitivity at the position of other uncorrelated sources, based on the information contained in $\boldsymbol{C}_{\mathbf{Y}}$ [9]. Note that the EIT-SF method presented here is fully compatible with the complete electrode model, as it only modifies the Jacobian matrix. This matrix is calculated only once, before proceeding with the SF.

\subsection{Activation index and covariance matrices}

The sensitivity of the LCMV filter decreases for increasing distance to the electrodes, so an absolute measurement is not appropriate. Instead, we define an index that normalizes the conductivity change contribution with respect to the noise only output. This is done based on the neural activity index $[10,12]$, defining a conductivity change index $\left(C C I_{k}\right)$ for each position $k$ as

$$
C C I_{k}=\frac{\boldsymbol{w}_{k}^{T} \boldsymbol{C}_{\mathrm{a}} \boldsymbol{w}_{k}}{\boldsymbol{w}_{k}^{T} \boldsymbol{C}_{\mathrm{n}} \boldsymbol{w}_{k}} .
$$

$\boldsymbol{C}_{\mathrm{a}}$ is the covariance matrix of the signal when the conductivity change of interest is present, and $\boldsymbol{C}_{\mathrm{n}}$ is the noise covariance matrix. The $C C I_{k}$ can be thought as the variance of the output of the distortionless response filter of (8), normalized by the variance of the output when only noise is present. Replacing (7) into (9), we obtain

$$
C C I_{k}=\frac{\boldsymbol{j}_{k}^{T} \boldsymbol{C}_{\mathbf{Y}}^{-1} \boldsymbol{C}_{\mathrm{a}} \boldsymbol{C}_{\mathbf{Y}}^{-1} \boldsymbol{j}_{k}}{\boldsymbol{j}_{k}^{T} \boldsymbol{C}_{\mathbf{Y}}{ }^{-1} \boldsymbol{C}_{\mathrm{n}} \boldsymbol{C}_{\mathbf{Y}}^{-1} \boldsymbol{j}_{k}},
$$

which is equivalent to the unit-noise-gain constraint variation of the LCMV filter, when $\boldsymbol{C}_{\mathrm{n}}$ is the identity matrix [9]. The $C C I_{k}$ is computed for all $K$ elements resulting in a conductivity change map, which we called the CCI map.

There exist several methods to estimate the covariance matrices $\left(\boldsymbol{C}_{\mathrm{a}}, \boldsymbol{C}_{\mathrm{n}}, \boldsymbol{C}_{\mathbf{Y}}\right)$, an important issue in SF [10]. The most often used estimator for $\boldsymbol{C}_{\mathbf{Y}}$ is the sample covariance matrix $\boldsymbol{C}$

$$
C=\frac{1}{T-1}(\boldsymbol{Y}-\overline{\boldsymbol{y}})(\boldsymbol{Y}-\overline{\boldsymbol{y}})^{T}
$$


where $\overline{\boldsymbol{y}}$ is the time average of $\boldsymbol{Y}$, and $T$ is the total number of snapshots. Note that in (7), we need the inverse of $\boldsymbol{C}_{\mathbf{Y}}$; however, especially with low $T, \boldsymbol{C}_{\mathbf{Y}}$ is ill-conditioned. Tikhonov regularization, also known as diagonal loading, is frequently applied to avoid this using $\boldsymbol{C}_{\mathbf{Y}}=\boldsymbol{C}+\alpha \boldsymbol{I}$, where $\alpha$ is the regularization parameter, and $\boldsymbol{I}$ is the identity matrix. Other regularization approaches particularly suited for $T<M(L-2)$ could also be used [30].

For the signal covariance matrix, we adopt the high signal-to-noise ratio approximation $\boldsymbol{C}_{\mathrm{a}}=\boldsymbol{C}$, which is common practice. The noise covariance matrix $\boldsymbol{C}_{\mathrm{n}}$ is typically estimated in a no signal condition or chosen as the identity matrix scaled by the noise variance if spatially stationary WGN is assumed as in our case.

\subsection{Linearly constrained minimum variance variants}

We summarize two possible variations for the LCMV filter: adding fixed-zero gain constraints and using a priori knowledge of the conductivity change time-course.

A known drawback of LCMV filters is that they may fail to separate correlated sources depending on their degree of correlation and their spatial distance. This effect can be reduced forcing zero gain constraints to particular regions that contain this kind of interference. These regions must be known or estimated a priori. The zero gain constraints are incorporated as in other spatial filters. Assume that the zero gain constraints are to be applied at positions $k 1, k 2, \ldots, k l$, and that $\boldsymbol{j}_{k 1}, \boldsymbol{j}_{k 2}, \ldots, \boldsymbol{j}_{k l}$ are the corresponding sensitivity vectors. The new weight vector $\boldsymbol{w}^{\prime}{ }_{k}$ with zero gain constraints at the specified positions is

$$
\boldsymbol{w}_{k}^{\prime T}=\boldsymbol{g}^{T}\left(\boldsymbol{J}_{l}^{T} \boldsymbol{C}_{\mathbf{Y}}^{-1} \boldsymbol{J}_{l}\right)^{-1} \boldsymbol{J}_{l}^{T} \boldsymbol{C}_{\mathbf{Y}}^{-1},
$$

where $\boldsymbol{J}_{l}=\left[\boldsymbol{j}_{k}, \boldsymbol{j}_{k 1}, \boldsymbol{j}_{k 2}, \ldots, \boldsymbol{j}_{k l}\right]$, and $\boldsymbol{g}=[1,0,0, \ldots, 0]^{T}$. The cost of incorporating zero gain constraints is that the output signal-to-noise ratio decreases and that $\boldsymbol{J}_{l}^{T} \boldsymbol{C}_{\mathbf{Y}}{ }^{-1} \boldsymbol{J}_{l}$ may become singular [9].

In some applications, the conductivity change time-course may be known from previous experiments. For example, a preliminary study with anesthetized rabbits showed that the conductivity in an induced ischemic stroke decreased almost linearly [31]. Knowledge of the time-courses is of help to distinguish a particular conductivity change of interest. Indeed, assuming that the normalized known waveform of a conductivity change of interest is $s(t)$ and that the vector $s$ contains the time samples of $s(t), \delta \sigma_{k}=\delta \sigma_{k} s$ where only the factor $\delta \sigma_{k}$ is unknown. One possible estimator is easily derived from (8) as

$$
\widehat{\delta \sigma_{k}}=\frac{{\widehat{\delta \sigma_{k}}}^{T} s}{\|\boldsymbol{s}\|^{2}}=\frac{\boldsymbol{w}_{k}^{T} \boldsymbol{Y} \boldsymbol{s}}{\|\boldsymbol{s}\|^{2}}
$$

Then, the $C C I_{k}$ index with known conductivity change evolution becomes

$$
C C I_{k}=\frac{\boldsymbol{j}_{k}^{T} C_{\mathbf{Y}}^{-1} C_{\mathbf{s}} C_{\mathbf{Y}}^{-1} j_{k}}{\boldsymbol{j}_{k}^{T} C_{\mathbf{Y}}{ }^{-1} C_{\mathrm{n}} C_{\mathbf{Y}}^{-1} j_{k}},
$$

where $C_{\mathrm{s}}=\frac{1}{T-1}(\boldsymbol{Y}-\overline{\boldsymbol{y}}) \boldsymbol{s} \boldsymbol{s}^{T}(\boldsymbol{Y}-\overline{\boldsymbol{y}})^{T}$.

\section{RESULTS}

Experiments were performed with simulations and with a cylindrical phantom providing real EIT signals. The simulations mimicked tank phantom experiments to assess the quality of our assumptions. In all experiments, we adopted $\boldsymbol{C}_{\mathrm{a}}=\boldsymbol{C}$, defining $\boldsymbol{C}$ as in (11), and $\boldsymbol{C}_{\mathrm{Y}}=\boldsymbol{C}+\alpha \boldsymbol{I}$. $\boldsymbol{C}_{\mathrm{n}}$ was chosen as the identity matrix. The injected current in the simulations and real experiments was set to $100 \mu \mathrm{V}$ peak. 
The CCI maps were computed to study bias and spatial resolution, and the filter output to study the evolution of conductivity changes. We compare the localization performance of the method with the GN-Laplace reconstruction of the form

$$
\delta \sigma_{t}^{\mathrm{GN}}=\left(\boldsymbol{J}^{T} \boldsymbol{J}+\lambda^{2} \boldsymbol{P}^{T} \boldsymbol{P}\right)^{-1} \boldsymbol{J}^{T} \boldsymbol{y}_{t} .
$$

$\boldsymbol{P}$ is the Laplacian discrete filter, and $\lambda$ is the regularization parameter, which was selected ad hoc for optimum performance. Note that using the Woodbury matrix inversion lemma, formulation (15) is equivalent to the Wiener filter:

$$
\delta \sigma_{t}^{\text {Wiener }}=\Sigma_{m} J^{T}\left(\boldsymbol{J} \Sigma_{m} J^{T}+C_{\mathrm{n}}\right)^{-1} \boldsymbol{y}_{t},
$$

with the smoothing prior $\left(\boldsymbol{P}^{T} \boldsymbol{P}\right)^{-1}$ as the source covariance matrix $\boldsymbol{\Sigma}_{m}$, and $\boldsymbol{C}_{\mathrm{n}}=\lambda^{2} \boldsymbol{I}$.

The GN-Laplace method results in an image per time frame, so we used for comparison the square of the root mean square $(R M S)$ of the $T$ individual images. Note that the CCI and the $R M S^{2}$ are both quadratic.

We calculated the PE and the RES metrics to quantify bias (or position error) and spatial resolution, respectively [14]. The PE was obtained as the Euclidean distance between the true center of gravity $(\mathrm{CoG})$ and the $\mathrm{CoG}$ of the resulting image. For one-source experiments, we selected the $q=1, \ldots, Q$ elements with a normalized CCI or normalized $R M S^{2}$ value higher than 0.95 , and we determined the $\mathrm{CoG}$ as

$$
\mathrm{CoG}=\frac{\sum_{q=1}^{Q} c_{q} x_{q}}{\sum_{q=1}^{Q} x_{q}},
$$

where $c_{q}$ and $x_{q}$ are the centroid and image value of element $q$, respectively. We defined the RES metric with a threshold of 0.75 :

$$
\operatorname{RES}=\sqrt{\frac{Q}{K}} .
$$

Note that a high value of the RES metric indicates low resolution and vice versa. For two sources, the images were divided in two regions by a vertical plane, as shown in Figure 1(c), and the PE and RES of each source were computed independently for each region. Table I shows the resulting PE, and Table II shows the resulting RES for the SF and GN-Laplace methods. The same mesh was used for computing all CCI maps (inverse problem mesh), but it differed from those used to generate the simulated signals to avoid any possible unwanted correlation induced by using the same mesh. The inverse problem mesh, shown in Figure 2(a), was composed of approximately 240000 tetrahedrons, with nodes at the electrode positions. All meshes were built with the aid of the ISO2MESH package [32].
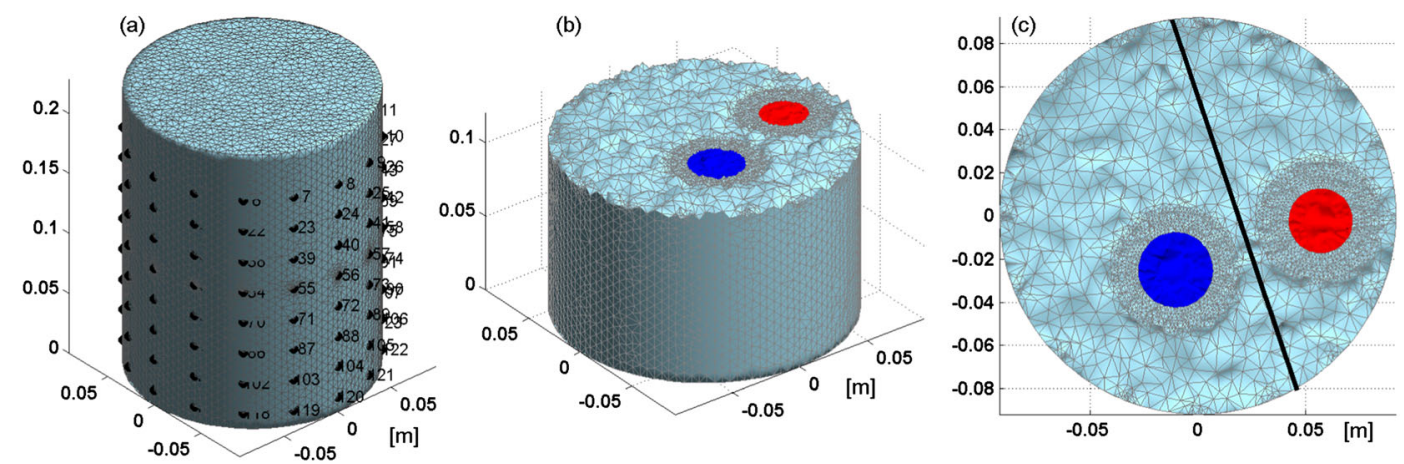

Figure 1. (a) Cylindrical phantom used to obtain electrical impedance tomography spatial filtering and Gaussian noise solutions; the dots ' $\bullet$ ' indicate the sensor positions. (b-c) Cylindrical phantom used to generate the simulated signals, sliced at $z=11 \mathrm{~cm}$. The two spherical regions with dynamic conductivity changes are colored with red (border) and blue (central). The straight line in (c) indicates the vertical plane that separate the volume in two regions to compute the metrics of each source. 
Table I. PE metric [mm].

\begin{tabular}{lcccccccc}
\hline & \multicolumn{3}{c}{ Simulated data } & \multicolumn{4}{c}{ Real data } \\
& \multicolumn{2}{c}{ One source } & \multicolumn{2}{c}{ Two sources } & \multicolumn{2}{c}{ One source } & Two sources \\
& Border & Central & Border & Central & Border & Central & Border & Central \\
\hline EIT-SF & 3.89 & 2.05 & 4.42 & 2.15 & 8.51 & 7.28 & $N / D^{\mathrm{a}}$ & 6.50 \\
GN-Laplace & 10.10 & 4.40 & 7.94 & $N / D^{\mathrm{a}}$ & 12.10 & 7.71 & 10.80 & $N / D^{\mathrm{a}}$ \\
\hline
\end{tabular}

a The CoG lies in the middle plane between the two sources.

EIT-SF, electrical impedance tomography spatial filtering; GN, Gauss-Newton.

Table II. RES metric $\left[\times 10^{-4}\right]$.

\begin{tabular}{lrrrrrrrr}
\hline & \multicolumn{4}{c}{ Simulated data } & \multicolumn{4}{c}{ Real data } \\
& \multicolumn{2}{c}{ One source } & \multicolumn{2}{c}{ Two sources } & \multicolumn{2}{c}{ One source } & Two sources \\
& Border & Central & Border & Central & Border & Central & Border & Central \\
\hline EIT-SF & 2.10 & 3.67 & 5.30 & 4.88 & 5.55 & 23.80 & $N / D^{\mathrm{a}}$ & 17.20 \\
GN-Laplace & 33.60 & 73.70 & 32.70 & $N / D^{\mathrm{a}}$ & 35.20 & 63.60 & 39.60 & $N / D^{\mathrm{a}}$ \\
\hline
\end{tabular}

a The CoG lies in the middle plane between the two sources.

EIT-SF, electrical impedance tomography spatial filtering; GN, Gauss-Newton.
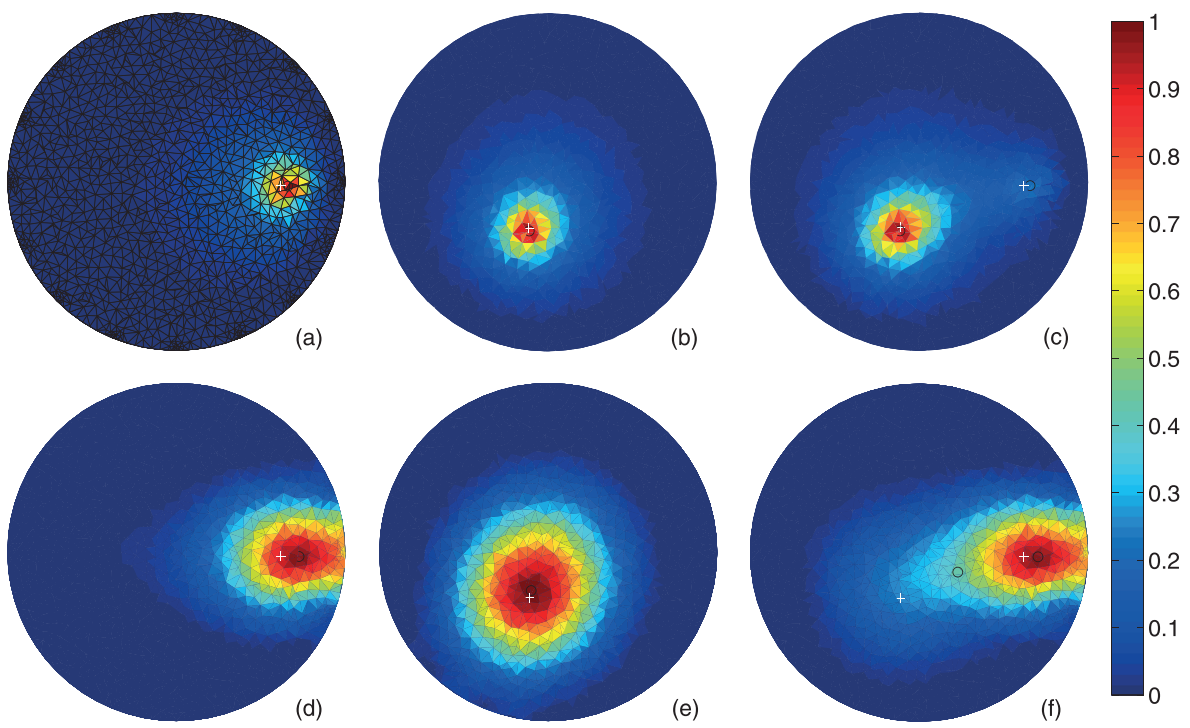

Figure 2. Simulation maps sliced at $z=11 \mathrm{~cm}$. (a-c) Normalized conductivity change index maps obtained with (a) the border source, (b) the central source, and (c) both simultaneous sources. (d-f) Gauss-Newton reconstruction with Laplacian prior, for (d) the border source, (e) the central source, and (f) both simultaneous sources. White crosses ' + ' indicate the true central position of the conductivity changes, and dark circles ' $\circ$ ' indicate the computed center of gravity.

\subsection{Simulated phantoms}

We generated a finite element model of a cylindrical $(9.2 \mathrm{~cm}$ radius and $22.85 \mathrm{~cm}$ height $) 3 \mathrm{D}$ phantom with 128 electrodes at its boundary, distributed evenly in eight rings of 16 electrodes each. We assumed a constant $1.75 \mathrm{~S} / \mathrm{m}$ baseline conductivity. Dynamic conductivity changes were assigned to one or two spherical regions (sources) of $3.5 \mathrm{~cm}$ diameter each, as shown in Figure 1. We simulated the signals at the electrode sites for 88 independent combinations of current injection pairs, resulting in a total of 88 combinations of 126 signals each. Figure 1 also shows the electrode positions and the mesh used to generate the signals with FEM. We generated 15 time frames or snapshots for all simulated signals, resulting in a $11088 \times 15$ data matrix $\boldsymbol{Y}$. We added some WGN with standard 
deviation $\sigma_{w}=0.2 \mu \mathrm{V}$, acting as electronic noise due to amplifiers. This value of $\sigma_{w}$ was estimated from the real tank measurements.

3.1.1. One localized conductivity change. We simulated sinusoidal conductivity changes varying between $1.75 \mathrm{~S} / \mathrm{m}$ (baseline conductivity) and $0.08 \mathrm{~S} / \mathrm{m}$ (conductivity of the objects used in the real experiments) at two different positions (expressed in cylindrical coordinates $(r[\mathrm{~cm}], \theta[\mathrm{rad}], z[\mathrm{~cm}])):(5.71,1.987 \pi, 11)$, labeled as border, and $(2.71,1.375 \pi, 11)$, labeled as central. Figure 2 depicts the normalized CCI maps and the reconstructed image using the GNLaplace method. Figure 3 shows the normalized filter outputs. The regularization parameter value was set following the asymmetric diagonal loading approach [9]. A value between the largest and the second largest eigenvalues of $\boldsymbol{C}_{\mathrm{Y}}, \lambda_{1}$, and $\lambda_{2}$ was adopted for the regularization parameter $\alpha_{n}$ of the $\boldsymbol{C}_{\mathbf{Y}}{ }^{-1}$ at the numerator of (10). A smaller value $\alpha_{d} \ll \lambda_{2}$ was adopted for the $\boldsymbol{C}_{\mathbf{Y}}{ }^{-1}$ at the denominator of (10).

3.1.2. Sensor position mismatch. We analyzed and compared the performance of the EIT-SF and the GN-Laplace methods randomly moving the electrode positions. We generated 10 different data sets varying the electrode positions randomly as a normal distribution with standard deviation of $1 \mathrm{~mm}$ in both height and azimuth. Figure 4 shows the 10 sets of electrodes and the resulting CoG with both methods and for both sources.
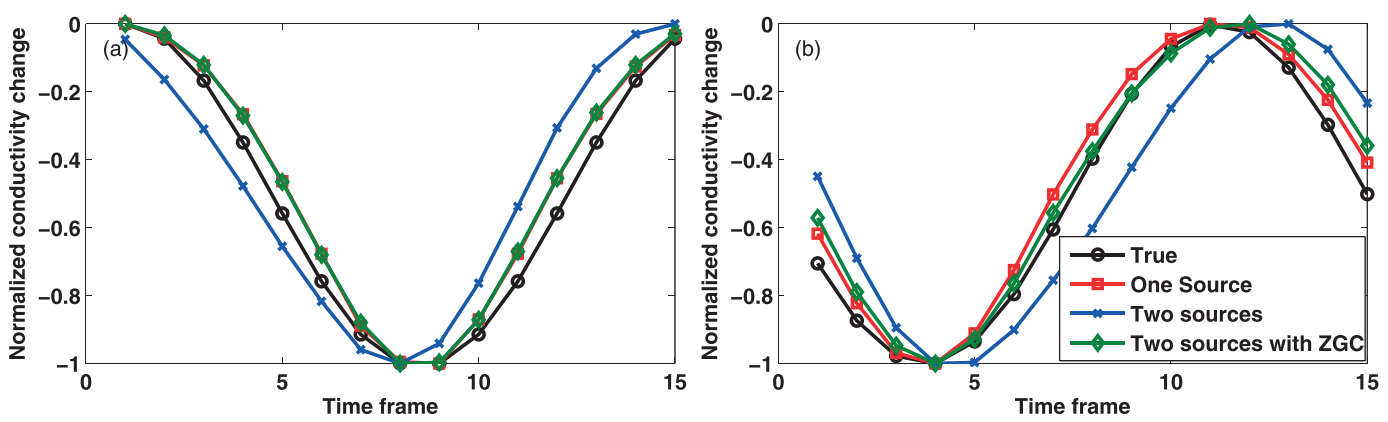

Figure 3. Normalized simulation outputs or time-courses for the (a) border conductivity change and the (b) central conductivity change. ZGC, zero gain constraints.
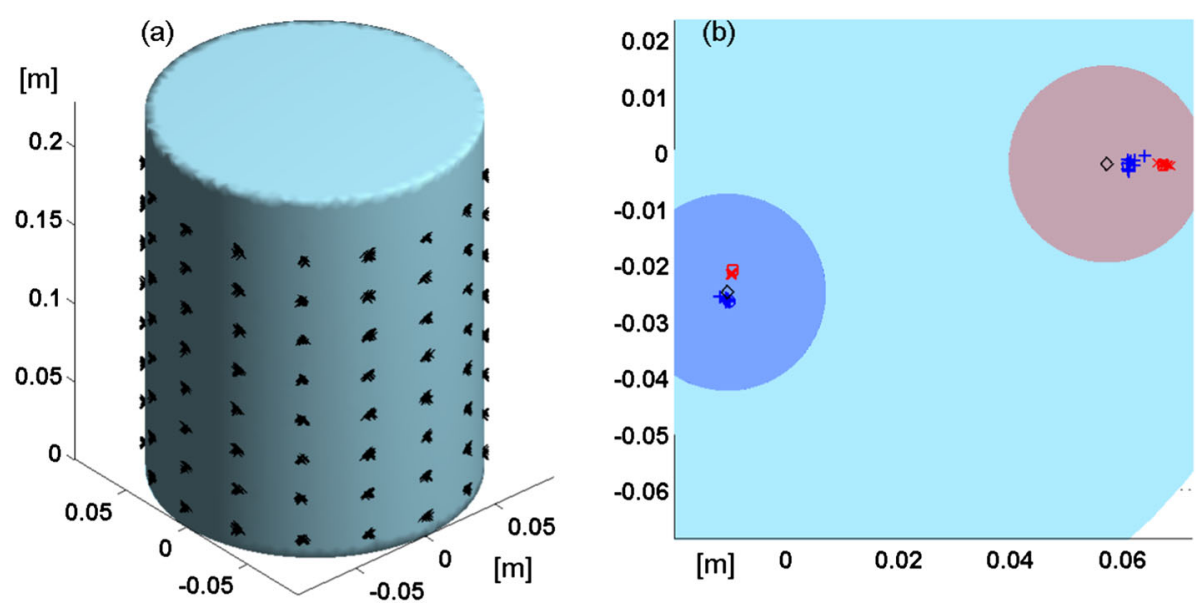

Figure 4. Simulations with noise in the sensor positions. (a) Electrode position samples. (b) Resulting center of gravity obtained with spatial filtering (blue '+') and with Gauss-Newton reconstruction with Laplacian prior (red ' $X$ '). The circles ' $O$ ' and squares ' $\square$ ' indicate the center of gravity obtained with spatial filtering and with Gauss-Newton reconstruction with Laplacian prior, respectively, when there is no error in the sensor positions. The diamonds ' $\diamond$ ' indicate the true central positions of the conductivity changes. 
3.1.3. Two localized conductivity changes. We simulated two simultaneous sources assigning two sinusoids with different phase to the central (blue), and to the border (red) regions of Figure 1(b, c). The obtained CCI map is depicted in Figure 2(c), together with the reconstructed images obtained with the GN-Laplace method (Figure 2(f)). Figure 5(a, b) shows the CCI maps obtained with seven zero gain constraints at each source position. The mesh is not shown for clarity. The regularization parameter values were chosen in a similar way as when simulating only one conductivity change. The only difference was that the numerator regularization parameter used to compute the CCI maps was set between the second and third largest eigenvalues of $\boldsymbol{C}_{\mathbf{Y}}$. The outputs of the filter for the central elements of each region are displayed in Figure 3.

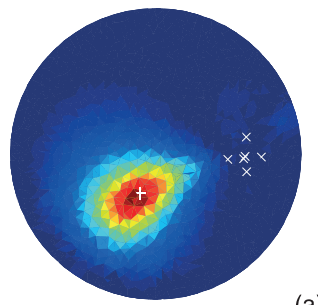

(a)

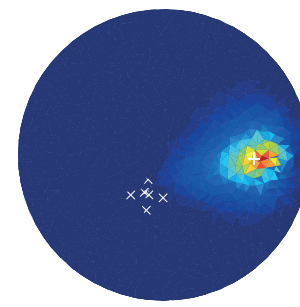

(b)

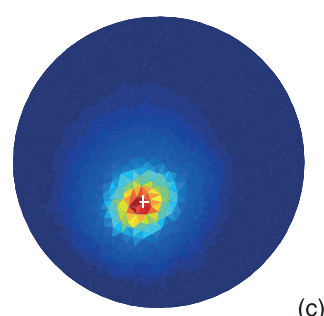

(c)

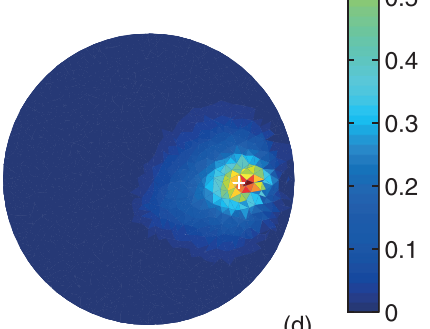

(d)

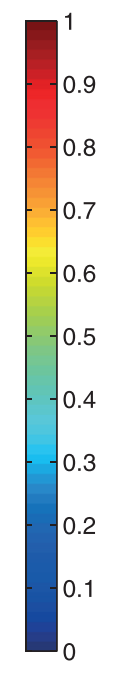

Figure 5. Linearly constrained minimum variance spatial filter variants for the simulations. Conductivity change index maps sliced at $z=11 \mathrm{~cm}$ : $(\mathrm{a}-\mathrm{b})$ with zero gain constraints and (c-d) with known conductivity change time-courses. The white crosses ' + ' indicate the true central position of the conductivity changes, and the white crosses ' $x$ ' indicate the position of zero gain constraints.
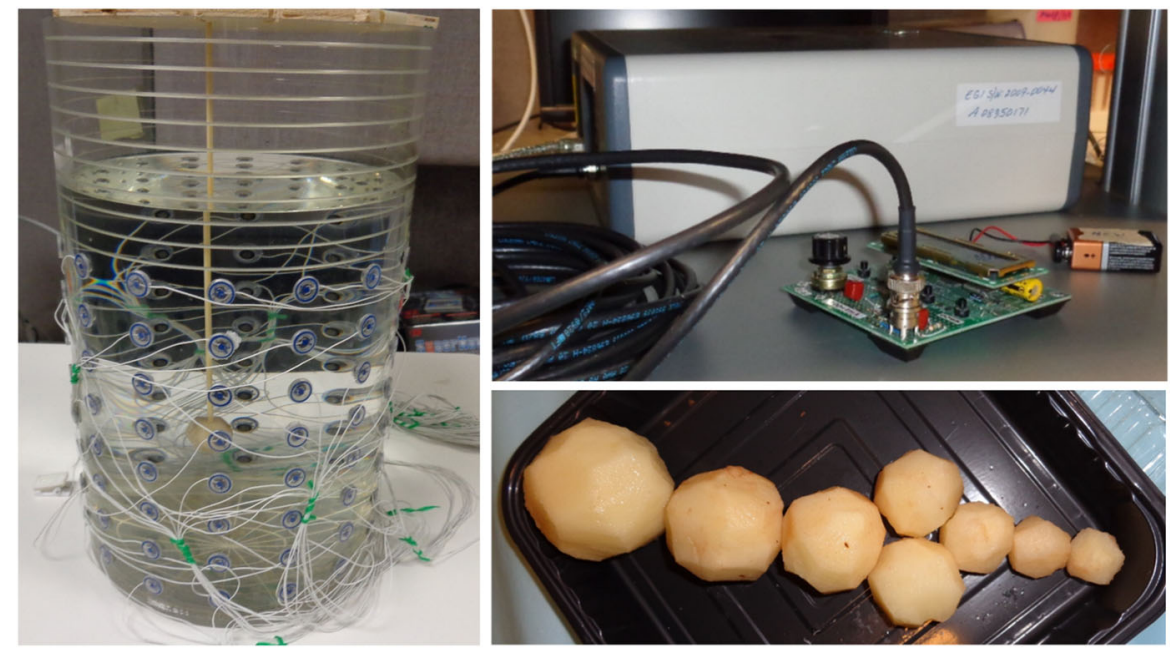

Figure 6. Left: cylindrical phantom used in the experiments. Top right: experimental electrical impedance tomography setup with the external current source used to collect the data. Bottom right: potatoes used in the experiments. 


\subsection{Real phantoms}

We tested the LCMV filter with real EIT signals measured in a cylindrical tank $(9.2 \mathrm{~cm}$ radius and $22.85 \mathrm{~cm}$ height) filled with saline (conductivity $1.75 \mathrm{~S} / \mathrm{m}$ ). We used potatoes (conductivity $0.08 \mathrm{~S} / \mathrm{m}$ [33]) of different sizes, shown in Figure 6, as the objects perturbing the saline bulk conductivity. The objects were held with a wooden stick at two different positions, at approximate coordinates $(5.71,1.987 \pi, 11)$ for the border and $(2.71,1.375 \pi, 11)$ for the central positions (expressed in cylindrical coordinates $(r[\mathrm{~cm}], \theta[\mathrm{rad}], z[\mathrm{~cm}]))$. Note that the positions are the same for both real and simulated experiments. We used seven different objects of approximate diameters of 2, 2.5, 3, 3.5, 4, 4.5, and $5 \mathrm{~cm}$. Data were collected with an experimental low-frequency EIT setup, developed by the Electrical Geodesics Inc., Eugene, Oregon, US. The EIT system prototype was based on a 256-channels commercial EEG system complimented by the current injection module and lock-in detection software. The phantom design, instrumentation, and data acquisition are described in details elsewhere [34], and the setup for this particular experiment is also shown in Figure 6. The acrylic cylinder tank was made approximately of the size of the typical adult human head. 128 standard EEG $\mathrm{Ag} / \mathrm{AgCl}$ electrodes with rubber o-rings were hermetically placed into the holes uniformly arranged on the lateral surface in eight rows and 16 columns. The electrodes were wired to the EEG system through the standard EEG leads (Electrical Geodesics, Inc.). Measurements were performed for 88 current injection pairs at current levels of $100 \mu \mathrm{V}$ (peak) and frequency $27 \mathrm{~Hz}$. The current injection protocol involved opposite electrodes in the same or adjacent rows,
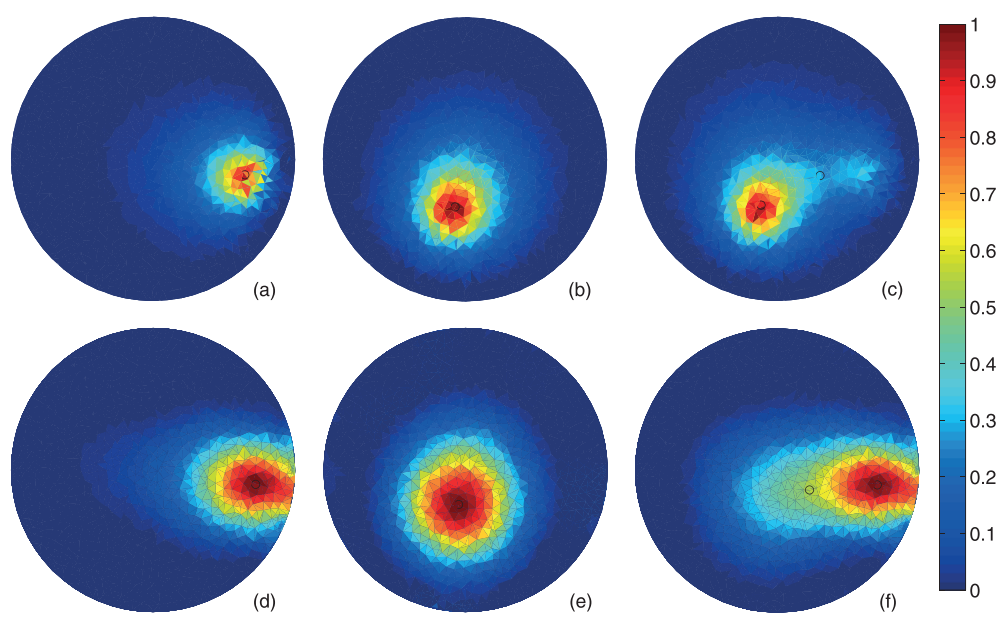

Figure 7. Real data maps sliced at $z=11 \mathrm{~cm}$. (a-c) Normalized conductivity change index maps obtained with (a) the border source, (b) the central source, and (c) both sources. (d-f) Gauss-Newton reconstruction with Laplacian prior for (d) the border source, (e) the central source, and (f) both sources simultaneously.

The dark circles ' $\circ$ ' indicate the computed center of gravity.
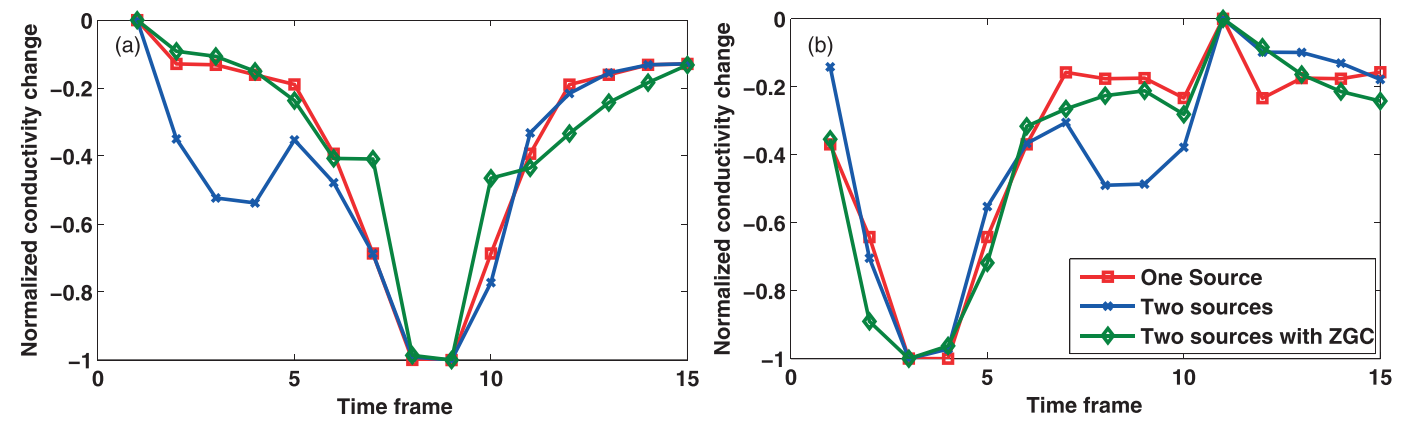

Figure 8. Normalized real data experiment outputs or time-courses for the (a) border conductivity change and the (b) central conductivity change. ZGC, zero gain constraints. 
generating a total of 88 sets of 126 potential measurements per object size and position. The built-in lock-in amplifier allowed to extract amplitude and phase of the raw EIT signals [35]. For each set of measurements, we obtained four samples of amplitude and phase by averaging the recording epochs of $4 \mathrm{~s}$ for each pair within a 1-s sliding window.

3.2.1. One localized conductivity change. We built two sinusoidal-like time-courses by sorting the potato measurements in the following orders $0,1,2,3,4,5,6,7,7,6,5,4,3,2,1$ and $5,6,7,7,6,5,4,3,2,1,0,1,2,3,4$ for border and central positions, respectively. Here, ' 0 ' means no object, ' 1 ' means the smallest object of $2 \mathrm{~cm}$ of diameter, and ' 7 ' means the largest object of $5 \mathrm{~cm}$ of diameter. Within each position, we used different measurement samples for same object sizes. The CCI maps are shown in Figure 7. The output conductivity changes are shown for both positions in Figure 8.

3.2.2. Two localized conductivity changes. Although the phenomenon is nonlinear for conductivity changes at different positions, we performed other simulations mimicking two simultaneous

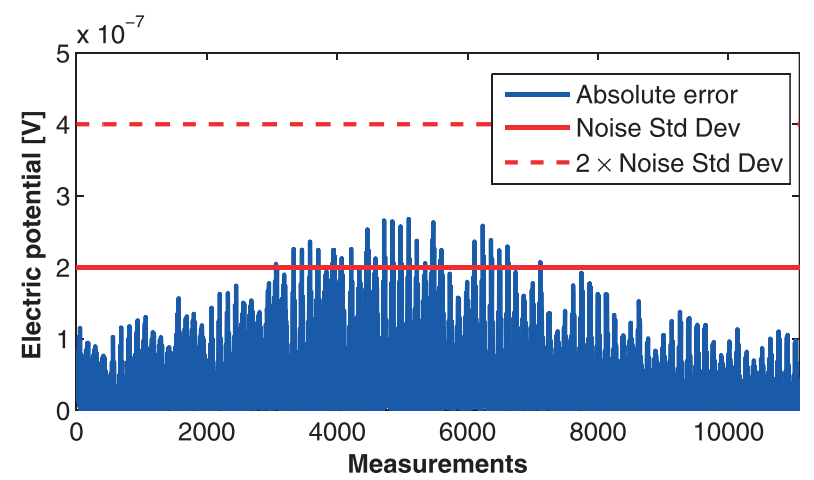

Figure 9. For building the two sources data set, we assumed linearity in the problem. The blue line shows the error made by doing this assumption in an equivalent simulated problem. The error is always lower than two times the noise standard deviation (Std Dev) of the real measurements.

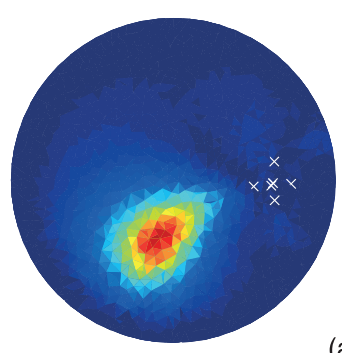

(a)

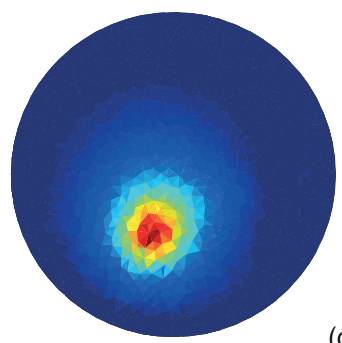

(c)

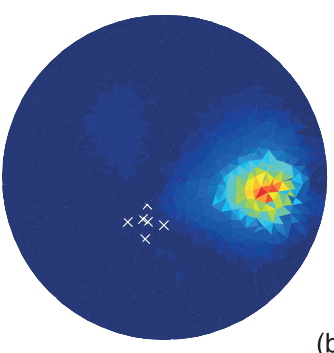

(b)

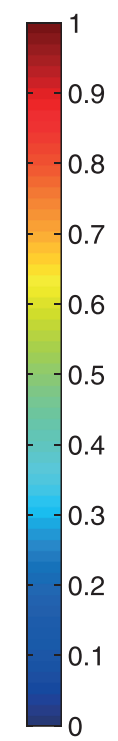

Figure 10. Linearly constrained minimum variance spatial filter variants for the real data experiments. Conductivity change index maps sliced at $z=11 \mathrm{~cm}$ : $(\mathrm{a}-\mathrm{b})$ with zero gain constraints and (c-d) with known conductivity change time-courses. The crosses ' $x$ ' indicate the position of zero gain constraints. 
objects at different positions and with varying sizes according to the individual real experiments of Section 3.2.1. We obtained two data sets, one with the two simultaneous objects (nonlinear) and another simply adding the two data sets obtained for each varying object at each position (linear assumption). We found that the effect of assuming linearity was lower than the noise level of the measurements. Explicitly, the maximum difference between nonlinear simulations and the linear assumption for all $88 \times 11,000=968,000$ simulated measurements was $0.25 \mu \mathrm{V}$, less than two times the noise standard deviation, as seen in Figure 9. This indicates that even if not negligible from a mathematical point of view, the effect of a linear approximation is of no practical significance in this particular setup. So, even though no two simultaneous objects were placed together in the tank for the real experiments, based on the previous analysis, we built the combined data set by adding together the two raw data sets corresponding to the single object measurements at two different positions.

Figure 7(c, e) depicts the maps obtained with SF and with GN-Laplace. Figure 10 depicts the CCI maps with seven zero gain constraints at the source positions and with the estimator of (14), assuming known conductivity change time-courses. These time-courses were obtained from the single source experiments. In Figure 8, we also show the outputs of the filter at the central position of each source.

\section{DISCUSSION}

\subsection{Source localization bias and resolution}

In Figure 2(a, b) and Table II, it is observed that the EIT-SF technique can be used to successfully localize with no bias and good resolution a localized conductivity change. Although the mathematical model is equivalent to that of an EEG source localization problem, we believe that the use of SF to localize conductivity changes is not as intuitive. Until now, SF was mostly associated with problems where real electromagnetic sources exist as in communications or in EEG/MEG.

For the real one-source data sets, we also show that the PE is lower with the LCMV filter than with GN-Laplace. Note that the error is slightly larger than in the simulations, which we consider is due to the irregular shape of the objects and to small uncertainties in the positioning of the objects. The spatial resolution was worse than in simulations because of the larger size of the objects compared with the simulated sources.

For two simulated regions with dynamic conductivity changes, Figure 2(c) shows that the algorithm could accurately localize the central position of the two varying conductivity regions with low bias, although the border source is barely seen. For two real conductivity changes, the peak of the CCI map in Figure 7(c) coincides with the central source position of Figure 7(b), and the border source is obscured.

Because in this work we are interested in localization of the conductivity changes but not in shape reconstruction, we compared the proposed method with the GN-Laplace reconstructor using only error metrics related to localization performance. Table I shows that the PE is always lower with the EIF-SF method than with the GN-Laplace method in both the simulated and real data. For the real experiments, we determined the central positions based on distance and angular measurements of the tank, the wooden stick, and the top cover, in the physical phantom and in the pictures. The RES of EIT-SF is always better than GN-Laplace, as can be seen on Table II.

The value of the regularization parameter is relevant when computing the CCI maps. The diagonal loading approach performed as expected in both simulated and real experiments. Note that in the two sources scenario, one source is not clearly visible. However, as discussed later, the use of zero gain constraints or the knowledge of the conductivity change time-course can be used to detect other sources.

\subsection{Conductivity change time-course}

Using the one-source simulated data set, the filter reconstructed the time-course of the conductivity change when pointing to the spatial position of maximum CCI, as seen in Figure 3. When using real data, the calculated time-courses of Figure 8 were coherent to what would be expected for changing 
objects. Except for the central source where the conductivity change of the smaller objects does not increase with the object sizes. However, the method still differentiates between a small object in the central region and nothing.

For two simultaneous conductivity changes with some correlation between them, the leakage distorts the output of the filter, as it is seen in Figure 3 and more clearly in Figure 8. The use of zero gain constraints reduces the effect of correlated interferences, as explained succeedingly.

\subsection{Zero gain constraints and knowledge of the time-course}

A known drawback of LCMV spatial filters is that they cannot separate fully correlated sources (i.e., modulus of the correlation coefficient equal to one), resulting in an activation map where the maximum is located between the sources [10]. If the sources are not fully correlated, the algorithm is able to separate them depending on the degree of correlation and on the spatial distance between them. The inclusion of zero gain constraints is a well-known technique to suppress correlated interference. Zero gain constraints are widely used in communications, where the direction of an interfering source may be fixed and known. Even though this possibility is mentioned in [9], we could not find in the literature the use of zero gain constraints with real signals in EEG or MEG. In the experiments, we show that this approach is fully applicable in EIT-SF. In Figures 5(a, b) and 10(a, b), it is observed that one conductivity change is more clearly visible with zero gain constraints at the position of the other source. In Figures 2(c) and 7(c), the border source is barely seen with both methods, but EIT-SF has the possibility of using zero gain constraints to detect it. Moreover, Figures 8 and 3 show that the use of zero gain constraints reduces the leakage, improving the quality of the reconstructed time-courses. Note that the filter outputs with zero gain constraints are the most similar to the filter outputs of the one-source data sets. In Figures 5(c, d) and 10(c, d), we show that the prior knowledge of the time-course can also be used to detect particular conductivity changes.

In this work, we provide a simple method to improve the conductivity change detection when the time-course is previously known (13). However, other approaches could be used with this additional information. A previously known dictionary of time-courses could be used to better localize conductivity changes associated to specific pathologies. Such a dictionary should be built using EIT-SF in controlled experiments.

\subsection{Sensor position mismatch}

In our simulations adding noise in the sensor positions, we found that, as seen in Figure 4, in all cases, the CoG obtained with the LCMV spatial filter was closer to the true central position of the conductivity changes than the GN-Laplace method. However, the variance of the CoG obtained with the GN-Laplace method was lower than the variance obtained with the LCMV spatial filter. Sensor position mismatch may induce errors, but some methods to account for inaccurate sensor positions have been proposed [36, 37]. Also, methods to accurately estimate sensor positions have been developed [38].

\section{CONCLUSIONS}

We successfully localize conductivity changes using SF with simulated and real EIT measurements. As far as we know, this is the first application of SF with real EIT data. We showed that the method can estimate the central position of one or two predominant localized conductivity changes accurately and with good spatial resolution. The use of zero gain constraints or a priori knowledge of the conductivity change evolution was shown to facilitate the detection of weaker sources, increasing resolution, and separating correlated sources. We compared the method with the classic GaussNewton with Laplace prior, showing better performance in bias and resolution, but showing larger variance for sensor position errors. Also, EIT-SF uses only one lead-field vector at a time, instead of using the full Jacobian matrix as in conventional reconstruction methods. This becomes advantageous in memory requirements when the number of elements of the mesh is high as in detailed 3D modeling of the head, which may require millions of elements [39]. Moreover, our alternative 
method to compute the Jacobian (Appendix A) may also be useful in such cases saving computational memory. We also described a straightforward procedure to select the regularization parameter value, which we consider an advantage of EIT-SF. We showed that the method can be also used to reconstruct the time-course of the conductivity at a particular region of interest. Because of the high temporal resolution of EIT and its portability, EIT-SF could provide new insights about conductivity dynamics.

In summary, we believe SF has advantages in localization problems, but other methods such as GN-Laplace or total variation [16] might be convenient in shape reconstruction problems. The results of this work illustrate the expected performance of EIT-SF in a general sense, not restricted to applications of EIT in the human brain. However, in a separate work, we study the performance of EIT-SF in particular applications of EIT to the brain using realistic head models [22]. We expect to test this method with real EIT measurements in animals or humans. So far, our results suggest that the use of EIT-SF is promising for early stroke characterization and for the localization of neuronal activity, where localized conductivity changes are expected.

\section{APPENDIX A: ALTERNATIVE SENSITIVITY MATRIX}

We describe a novel method, used in this work, to compute the sensitivity matrix $\boldsymbol{J}$ of (2). It is based on the mathematical equivalence between a localized conductivity change in EIT and a dipolar electromagnetic source in EEG [16].

Let $\sigma(\vec{x})$ be the conductivity at each point of the space $\vec{x}$ of a volume $\Omega$. When an electric current is applied, it produces an electric potential distribution $\Phi(\vec{x})$. A conductivity change $\delta \sigma(\vec{x})$ results in a change of the electric potential distribution $\delta \Phi(\vec{x})$. For frequencies low enough such that the quasistatic approximation of Maxwell equations holds, up to several kilohertz for the head tissues, the equation that governs the physics of the problem is

$$
\nabla \cdot(\sigma(\vec{x}) \nabla \Phi(\vec{x}))=0,
$$

in all $\vec{x}$ except from the current injection points. Eq. A.1 must also hold when the conductivity change is present:

$$
\nabla \cdot((\sigma(\vec{x})+\delta \sigma(\vec{x})) \nabla(\Phi(\vec{x})+\delta \Phi(\vec{x})))=0 .
$$

Applying properties of the gradient operator, (A.2) becomes

$$
\nabla \cdot(\sigma \nabla \Phi)+\nabla \cdot(\sigma \nabla \delta \Phi)+\nabla \cdot(\delta \sigma \nabla \Phi)+\nabla \cdot(\delta \sigma \nabla \delta \Phi)=0,
$$

where the dependence with $\vec{x}$ has been omitted for clarity. The first term of (A.3) is zero because of (A.1), and the last term in (A.3) can be neglected for relatively small conductivity changes as it is $\mathcal{O}\left(\delta \sigma^{2}\right)[16]$. Then, Eq. A.2 can be approximated by

$$
\nabla \cdot(\sigma \nabla \delta \Phi) \approx-\nabla \cdot(\delta \sigma \nabla \Phi) .
$$

This relationship is equivalent to the equation

$$
\nabla \cdot\left(\sigma \nabla \Phi_{\mathrm{EEG}}\right)=\nabla \cdot \vec{J}_{\mathrm{p}}
$$

which governs the EEG FP, where $\vec{J}_{\mathrm{p}}$ is the primary current density of a dipolar source, and $\Phi_{\mathrm{EEG}}$ is the resulting electric potential. The EEG FP is to estimate the potential at the $L$ electrodes when a dipole source is located at position $k$. Looking at (A.4) and (A.5), the difference EIT FP is equivalent to the EEG FP. The difference of the potential in EIT $\delta \Phi$ corresponds to $\Phi_{\mathrm{EEG}}$ and the source in EIT is minus the conductivity change multiplied by the gradient of the potential $(\delta \sigma \nabla \Phi)$.

In EEG, the signal model for a dipolar source $\boldsymbol{q}$ at position $k$ involves a lead field matrix $\boldsymbol{L} \boldsymbol{F}_{k}$, which is the expected electric potential at the electrodes generated by that source. This matrix has as many columns as dimensions used to formulate the problem: one column or lead field vector per canonical orientation of the dipolar source. The signal model is

$$
\boldsymbol{y}_{\mathrm{EEG}}=\boldsymbol{L} \boldsymbol{F}_{k} \boldsymbol{q}+\boldsymbol{n}(t)
$$


where $\boldsymbol{y}_{\mathrm{EEG}}$ is an $L$ measurement vector ( $L$ is the total number of electrodes) if assuming average reference for the measurements. There are some differences between the signal model of EEG (A.6) and the signal model of difference EIT (4). The EIT measurement vector $\boldsymbol{y}$ has $(L-2) M$ elements ( $M$ is the number of different current injection pairs used to measure), whereas $\boldsymbol{y}_{\mathrm{EEG}}$ has $L$. If we have $\boldsymbol{L} \boldsymbol{F}_{k}$, we can form a new matrix $\overline{\boldsymbol{L F}}_{k}^{m}$ for each current injection pair $m$ in the following way: the rows of $\boldsymbol{L} \boldsymbol{F}_{k}$ corresponding to the current injection electrodes are removed, and then the average of each column is also removed to maintain the average reference. Then, the expected $\delta \Phi$ at the electrodes in EIT is $\overline{\boldsymbol{L F}}_{k}^{m} \nabla \Phi_{k}^{m}$ when a conductivity change $\delta \sigma$ is present at position $k$, and the current injection pair is $m . \nabla \Phi_{k}^{m}$ is the gradient of the electric potential at $k$ for the current injection pair $m$. Arranging the $\overline{\boldsymbol{L F}}_{k}^{m} \nabla \Phi_{k}^{m}$ vectors for the $M$ current injection pairs, we can form each vector $\boldsymbol{j}_{k}$, that is, each column of $\boldsymbol{J}$.

For this work, we computed each $\boldsymbol{L} \boldsymbol{F}_{k}$ solving numerically the EEG FP using the FEM, and the gradient of the potential was estimated for the homogeneous tank model with a baseline conductivity value of $1.75 \mathrm{~S} / \mathrm{m}$. We compared the vectors $\boldsymbol{j}_{k}$ obtained with this method with the solutions of the EIT FP for a conductivity change at element $k$, and we found no appreciable differences.

\section{ACKNOWLEDGEMENTS}

This work was supported by the ANPCyT PICT 2011-11-0909, UNLP 11-I-166, CONICET, and CIC-pBA. Special thanks go to Sergei Turovets and Don Tucker for their help with the EIT experimental setup. The corresponding author would like to acknowledge support from the Fulbright Foundation, USA, and the Fundación Bunge \& Born, Argentina, which permitted a visit to the University of Oregon Neuroinformatics Center and Electrical Geodesics, Inc., Eugene, USA, where the EIT experiments in a real phantom were performed.

\section{REFERENCES}

1. Bayford R. Bioimpedance tomography (electrical impedance tomography). Annual Review of Biomededical Engineering 2006; 8:63-91.

2. Cherepenin V, Karpov A, Korjenevsky A, Kornienko V, Kultiasov Y, Ochapkin M, Trochanova O, Meister J. Threedimensional EIT imaging of breast tissues: system design and clinical testing. IEEE Transactions on Medical Imaging 2002; 21(6):662-667.

3. Clay M, Ferree T. Weighted regularization in electrical impedance tomography with applications to acute cerebral stroke. IEEE Transactions on Medical Imaging 2002; 21(6):629-638.

4. Bagshaw A, Liston A, Bayford R, Tizzard A, Gibson A, Tidswell A, Sparkes M, Dehghani H, Binnie C, Holder D. Validation of reconstruction algorithms for electrical impedance tomography of human brain function. NeuroImage 2003; 20:752-764

5. Fabrizi L, Horesh L, McEwan A, Holder D. A feasibility study for imaging of epileptic seizures by EIT using a realistic fem of the head. 14 ed. IFMBE, Vol. 14, Seoul, 2006; 3874-3877.

6. Abascal JFP, Arridge SR, Atkinson D, Horesh R, Fabrizi L, Lucia MD, Horesh L, Bayford RH, Holder DS. Use of anisotropic modelling in electrical impedance tomography; description of method and preliminary assessment of utility in imaging brain function in the adult human head. NeuroImage 2008; 43(2):258-268.

7. Holder D. Electrical impedance tomography of brain function. World Automation Congress, Hawaii, 2008; 1-6.

8. Gilad O, Holder D. Impedance changes recorded with scalp electrodes during visual evoked responses: implications for electrical impedance tomography of fast neural activity. NeuroImage 2009; 47:514-522.

9. Sekihara K, Nagarajan S S. Adaptive Spatial Filters for Electromagnetic Brain Imaging. Springer: Berlin, 2008.

10. van Been BD, van Drongelen W, Yuchtman M, Suzuki A. Localization of brain electrical activity via linearly constrained minimum variance spatial filtering. IEEE Transactions on Biomedical Engineering 1997; 44(9):867-880.

11. Vrba J, Robinson S. Linearly constrained minimum variance beamformers, synthetic aperture magnetometry, and MUSIC in MEG applications. Conference Record of the Thirty-Fourth Asilomar Conference on Signals, Systems and Computers, 2000, Vol. 1, Pacific Grove, California, 2000; 313-317.

12. Chen YS, Cheng CY, Hsieh JC, Chen LF. Maximum contrast beamformer for electromagnetic mapping of brain activity. IEEE Transactions on Biomedical Engineering 2006; 53(9):1765-1774.

13. Javaherian A, Movafeghi A, Faghihi R. Reducing negative effects of quadratic norm regularization on image reconstruction in electrical impedance tomography. Applied Mathematical Modelling 2013; 37(8):5637-5652.

14. Adler A, Arnold J, Bayford R, Borsic A, Brown B, Dixon P, Faes T, Frerichs I, Gagnon H, Gärber Y, Grychtol B, Hahn G, Lionheart W, Malik A, Patterson R, Stocks J, Tizzard A, Weiler N, Wolf G. GREIT: A unified approach to 2D linear EIT reconstruction of lung images. Physiological Measurement 2009; 30(6):S35-S55. 
15. Liu J, Lin L, Zhang W, Li G. A novel combined regularization algorithm of total variation and Tikhonov regularization for open electrical impedance tomography. Physiological Measurement 2013; 34(7):823-838.

16. Lionheart W, Polydorides N, Borsic A. Electrical Impedance Tomography: Methods, History and Applications, Holder DS (ed.)., chap. The reconstruction problem, Inst. Phys. Institute of Physics Publishing, Ltd.: UK, 2005; 3-64.

17. Nachman AI. Global uniqueness for a two-dimensional inverse boundary value problem. Annals of Mathematics 1996; 143(1):71-96.

18. Isaacson D, Mueller JL, Newell JC, Siltanen S. Reconstructions of chest phantoms by the D-bar method for electrical impedance tomography. IEEE Transactions on Medical Imaging 2004; 23(7):821-828.

19. Vauhkonen M, Karjalainen P, Kaipio J. A Kalman filter approach to track fast impedance changes in electrical impedance tomography. IEEE Transactions on Biomedical Engineering 1998; 45(4):486-493. DOI: $10.1109 / 10.664204$.

20. Adler A, Dai T, Lionheart WRB. Temporal image reconstruction in electrical impedance tomography. Physiological Measurement 2007; 28(7):S1-S11.

21. Fernández-Corazza M, von Ellenrieder N, Muravchik CH. Spatial filtering in electrical impedance tomography. Journal of Physics: Conference Series 2012; 407(1):1-6.

22. Fernández-Corazza M, von Ellenrieder N, Muravchik CH. EIT spatial filtering in realistically shaped head models. 15th Interational Conference on Biomedical Applications of Electrical Impedance Tomography, Gananoque, Ontario, Canada, 2014; 31-31.

23. Gonçalves S, de Munck J, Verbunt J, Bijma F, Heethaar R, da Silva FL. In vivo measurement of the brain and skul resistivities using an EIT-based method and realistic models for the head. IEEE Transactions on Biomededical Engineering 2003; 50(6):754-767.

24. Fernández-Corazza M, Beltrachini L, von Ellenrieder N, Muravchik $\mathrm{CH}$. Waveform selection for electrical impedance tomography. IEEE Latin America Transactions 2013; 11(1):402-407.

25. Fernández-Corazza M, von Ellenrieder N, Muravchik CH. Estimation of electrical conductivity of a layered spherical head model using electrical impedance tomography. Journal of Physics: Conference Series 2011; 332(1):012022.

26. Xu Z, He W, He C, Zhang Z, Liu Z. The analytical solution of EIT forward problem based on a multilayer spherical model. World Automation Congress, Hawaii, 2008; 1-5.

27. Kleinermann F, Avis N, Alhargan F. Analytical solution to the three-dimensional electrical forward problem for a circular cylinder. Inverse Problems 2000; 16(2):461-468.

28. Fernández-Corazza M, Beltrachini L, von Ellenrieder N, Muravchik C H. Tomografía de impedancia eléctrica y resonancia magnética como herramientas conjuntas para la estimación paramétrica de la conductividad eléctrica del cráneo y del cuero cabelludo. XIV Reunión de Trabajo Procesamiento de la Información y Control RPIC 2011, Oro Verde, 2011; 845-850.

29. Abascal JFPJ, Arridge SR, Lionheart WRB, Bayford RH, Holder DS. Validation of a finite-element solution for electrical impedance tomography in an anisotropic medium. Physiological Measurement 2007; 28(7):S129—S140.

30. Beltrachini L, von Ellenrieder N, Muravchik C. Shrinkage approach for spatiotemporal EEG covariance matrix estimation. IEEE Transactions on Signal Processing 2013; 61(7):1797-1808. DOI: 10.1109/TSP.2013.2238532.

31. Shi X, You F, Fu F, Liu R, Dai M, Dong X. Preliminary research on monitoring of cerebral ischemia using electrical impedance tomography technique. 30th IEEE EMBS Conference, Vancouver, 2008; 1188-1191.

32. Fang Q, Boas D A. Tetrahedral mesh generation from volumetric binary and gray-scale images. Proceedings of the Sixth IEEE International Conference on Symposium on Biomedical Imaging: From Nano to Macro, ISBI'09, IEEE Press: Piscataway, NJ, USA, 2009; 1142-1145.

33. Halden K, Alwis AAPD, Fryer PJ. Changes in the electrical conductivity of foods during ohmic heating. International Journal of Food Science \& Technology 1990; 25:9-25.

34. Esler B, Lyons T, Turovets S, Tucker D. Instrumentation for low frequency EIT studies of the human head and its validation in phantom experiments. Journal of Physics: Conference Series 2010; 224(1):1-4.

35. Poolman P, Frank R, Turovets S. Modified lock-in detection for extraction of impressed EEG signals in lowfrequency bounded-EIT studies of the human head. Congress on Image and Signal Processing, 2008. CISP 'O8, Vol. 1, Sanya, Hainan, China, 2008; 174-183.

36. Dard J, Hakula H, Hyvönen N, Staboulis S. Fine-tuning electrode information in electrical impedance tomography. Inverse Problems and Imaging 2012; 6(3):399-421.

37. Dai T, Gómez-Laberge C, Adler A. Reconstruction of conductivity changes and electrode movements based on EIT temporal sequences. Physiological Measurement 2008; 29(6):S77-S88.

38. von Ellenrieder N, Beltrachini L, Blenkmann A, Fernández-Corazza M, Kochen S, Muravchik C. A low-cost and robust photogrammetry method for determining electrode positions. 30th International Epilepsy Congress, Vol. 54, Montreal, Canada, 2013; 358-358.

39. Wolters C, Anwander A, Tricoche X, Weinstein D, Koch M, MacLeod R. Influence of tissue conductivity anisotropy on EEG/MEG field and return current computation in a realistic head model: a simulation and visualization study using high-resolution finite element modeling. NeuroImage 2006; 30(3):813-826. 